\title{
Abicipar pegol-a novel anti-VEGF therapy with a long duration of action
}

\author{
Elad Moisseiev ${ }^{1,2} \cdot$ Anat Loewenstein ${ }^{1,3}$
}

Received: 9 July 2019 / Accepted: 9 July 2019 / Published online: 19 September 2019

(c) The Royal College of Ophthalmologists 2019

Intravitreal injections have revolutionized the management of retinal diseases, and since their advent have rapidly become the most commonly performed procedure in ophthalmology. The majority of intravitreal injections include administration of anti-vascular endothelial growth factor (VEGF) agents, such as bevacizumab, ranibizumab, or aflibercept. VEGF has been found to be a key molecule in numerous retinal diseases, and its inhibition has been established as a highly effective treatment for these diseases, including the most common retinal conditionsneovascular age related macular degeneration (AMD), diabetic macular edema, and retinal vein occlusions.

Although intravitreal injections are very safe and effective, they require frequent monitoring and repeated visits for imaging, examination and injections, over a long period of time. Therefore, they constitute a significant burden on patients, ophthalmologists, and health systems. In order to address this issue, continued research efforts are being made toward developing longer-lasting anti-VEGF therapy, which will require less frequent visits and injections while maintaining their excellent therapeutic effect.

Available anti-VEGF agents are based on antibodies or their fragments. Recent advances in bioengineering technology have led to the development of designed ankyrin repeat proteins (DARPins), which mimic antibodies and can be designed to have high specificity and affinity to a selected target. They are smaller than antibodies and may therefore have better tissue penetration, have a high affinity

Elad Moisseiev

elad_moi@netvision.net.il

1 Sackler School of Medicine, Tel Aviv University, Tel Aviv, Israel

2 Department of Ophthalmology, Meir Medical Center, Kfar Saba, Israel

3 Department of Ophthalmology, Tel Aviv Medical Center, Tel Aviv, Israel that enables them to be active at lower concentrations, and are more stable so their effect may be longer lasting.

Abicipar pegol (Abicipar, Allergan. Dublin, Ireland) is a DARPin directed to bind all VEGF-A isoforms, similar to ranibizumab. It has a higher affinity and a longer intraocular half-life than ranibizumab (>13 days vs. 7.2 days) [1, 2], making it a potential anti-VEGF therapy with longer duration and need for less frequent injections.

The REACH study [3] was a phase II multicenter randomized controlled trial that compared abicipar $1 \mathrm{mg}$, abicipar $2 \mathrm{mg}$, and ranibizumab in patients with naïve neovascular AMD. The study included overall 64 patients that were followed for 20 weeks, in which patients treated with abicipar received three monthly injections and those treated with ranibizumab received five monthly injections. Best-corrected visual acuity (BCVA) change from baseline was $+6.2,+8.3$, and +5.6 letters at week 16 for abicipar 1 $\mathrm{mg}$, abicipar $2 \mathrm{mg}$, and ranibizumab, and $+8.2,+10.0$, and +5.3 letters at week 20 , respectively. Reduction in mean central retinal thickness (CRT) compared with baseline was 134,113 , and $131 \mu \mathrm{m}$ at week 16 for abicipar $1 \mathrm{mg}$, abicipar $2 \mathrm{mg}$, and ranibizumab, and 116,103 , and $138 \mu \mathrm{m}$ at week 20, respectively. Overall, BCVA and CRT improvements were similar in both abicipar groups to those achieved with ranibizumab, and were maintained for 3 months following the third abicipar injection.

These encouraging results paved the way to phase III studies on abicipar for the treatment of neovascular AMD. The SEQUOIA and CEDAR studies included patients with neovascular AMD divided into three arms: three monthly abicipar $2 \mathrm{mg}$ injections followed by an injection every 8 weeks, two monthly abicipar $2 \mathrm{mg}$ injections followed by an injection after 8 weeks and every 12 weeks thereafter, and monthly ranibizumab injections. The primary outcome measure was stable vision, defined as the proportion of patients with less than 15 letters loss of VA compared with baseline. The results of these studies reported high rates (over 90\%) of stable vision in all three arms. The proportion of patients with more than 15 letters of VA gain was similar 
between the abicipar and ranibizumab groups in SEQUOIA, but higher in the ranibizumab group in CEDAR. Overall, abicipar demonstrated non-inferiority compared with ranibizumab, with less frequent injections (https://www. molecularpartners.com/allergan-and-molecular-partners-a nnounce-two-positive-phase-3-clinical-trials-for-abiciparpegol-8-and-12-week-regimens-for-the-treatment-in-pa tients-with-neovascular-age-related-macular-degeneration/).

In addition, the results of two smaller phase II clinical trials comparing the efficacy of abicipar $1 \mathrm{mg}$ and $2 \mathrm{mg}$ to ranibizumab between Japanese and non-Japanese patients with neovascular AMD have recently been published. The BAMBOO (conducted in Japan) and CYPRESS (conducted in the US) studies included 25 patients each, divided into the same three treatments arms and injection schedules as in REACH. At the primary endpoint of 16 weeks, mean improvement in vision was 7.8 letters, 8.9 letters, and 17.4 letters for abicipar $1 \mathrm{mg}$, abicipar $2 \mathrm{mg}$, and ranibizumab in BAMBOO, and 4.4 letters, 10.1 letters, and 15.2 letters, respectively, in CYPRESS. Results with both dosages of abicipar were comparable between studies, indicating that its efficacy is similar in Japanese and non-Japanese patients [4]. The Japanese cohort in BAMBOO included patients with polypoidal choroidal vasculopathy (PCV), which often responds less favorably to ranibizumab.

Although in some of the abovementioned studies results were better in the monthly ranibizumab groups, abicipar has met the non-inferiority criteria in REACH and achieved good results in maintaining BCVA gains 8 to 12 months after the last injection. These results indicate that abicipar has the potential to become an extended-duration antiVEGF agent, which will possibly allow treating patients in intervals of 12 weeks and reduce the ever-increasing burden of anti-VEGF injections. However, this promise is troubled by a relatively high rate of intraocular inflammation (IOI; uveitis or vitritis) following abicipar injections. Rates of IOI were $10.4 \%$ in REACH, $15.3 \%$ in CEDAR\&SEQUOIA and $7.5 \%$ in BAMBOO\&CYPRESS, significantly higher than the near-zero rates with ranibizumab and other antiVEGF agents. It should be noted that most cases of IOI were mild, and severe IOI (incapacitating from regular activities and work) occurred $3.5 \%$ of abicipar treated patients in CEDAR\&SEQUOIA. To address this concern, the manufacturing process was modified to achieve a more purified product. In the MAPLE study, 123 neovascular AMD patients were randomized to treatment by the modified formulation of abicipar $2 \mathrm{mg}$ or sham. Overall rates of IOI were 8.9 , with only one case $(1.6 \%)$ of severe IOI (https://www.molecularpartners.com/allergan-and-molecula r-partners-announce-topline-safety-results-from-maplestudy-of-abicipar-pegol/).

In conclusion, DARPins are a promising technology and abicipar is in a relatively advanced stage of development and holds promise to become a long-acting effective antiVEGF therapy. Additional research and clinical trials are required to improve its safety and validate its efficacy and extended duration compared with the currently available anti-VEGF agents, and hopefully it will be added to the treatment arsenal and reduce the burden of intravitreal injections.

\section{Compliance with ethical standards}

Conflict of interest The authors declare that they have no conflict of interest.

Publisher's note Springer Nature remains neutral with regard to jurisdictional claims in published maps and institutional affiliations.

\section{References}

1. Krohne TU, Liu Z, Holz FG, Meyer CH. Intraocular pharmacokinetics of ranibizumab following a single intravitreal injection in humans. Am J Ophthalmol. 2012;154:682-6.

2. Rodrigues GA, Mason M, Christie LA, Hansen C, Hernandez LM, Burke J, et al. Functional characterization of abicipar-pegol, an Anti-VEGF DARPin therapeutic that potently inhibits angiogenesis and vascular permeability. Invest Ophthalmol Vis Sci. 2018; 59:5836-46.

3. Callanan D, Kunimoto D, Maturi RK, Patel SS, Staurenghi G, Wolf $S$, et al. Double-masked, randomized, phase 2 evaluation of abicipar pegol (an anti-VEGF DARPin Therapeutic) in neovascularage-related macular degeneration. J Ocul Pharmacol Ther. 2018.

4. Kunimoto D, Ohji M, Maturi RK, Sekiryu T, Wang Y, Pan G, et al. BAMBOO and CYPRESS Study Groups Evaluation of abicipar pegol (an Anti-VEGF DARPin Therapeutic) in patients with neovascular age-related macular degeneration: studies in Japan and the United States. Ophthalmic Surg Lasers Imaging Retin. 2019;50: e10-e22. 